\title{
La industria en la política planificadora de la Junta de Andalucía
}

\author{
Adolfo Rodero Franganillo'
}

En este artículo se estudia el tratamiento que la Junta de Andalucía ha dado al sector industrial en el conjunto de su política planificadora, utilizando para ello los planes elaborados por el gobierno andaluz. Ciertamente existen medidas de política industrial no incluidas en dichos documentos, pero dada la metodología que han seguido los redactores de los planes, podemos considerar que lo esencial de la política industrial forma parte de los programas económicos de la Junta ${ }^{2}$.

Antes de entrar en el análisis concreto de los planes, es indispensable recordar brevemente la situación y las características del sector industrial andaluz, a ello dedicaremos los apartados 1 a 5 de este artículo².

\section{La industria andaluza durante el período autonómico.}

Desde el inicio del periodo autonómico hasta la actualidad, se han producido diversos hechos de gran importancia para la economía andaluza:

$\left.1^{\circ}\right)$ La creación del estado de las autonomías, lo que ha permitido la puesta en vigor de una política económica andaluza, dentro de las limitaciones que marca la Constitución.

20) La adhesión de España a la entonces Comunidad Económica Europea, con importantes efectos, dentro de un marco económico mucho más amplio, sobre la política regional.

1. Profesor Titular de área de la Facultad de Ciencias de Ciencias Económicas y Empresariales-ETEA, Universidad de Córdoba.

2. Algunas de las ideas y de los comentarios contenidos en estos apartados fueron expuestos anteriormente en: A. RODERO (1996), La industria andaluza en el periodo autonómico, Revista de Estudios Regionales, $n^{2} 44$, pp. 307-320. 
$\left.3^{\mathbf{9}}\right)$ Una evolución económica marcada por etapas de distinto signo de uno o varios ciclos económicos.

Todos estos fenómenos han influido, sin duda, sobre la industria regional, con resultados que a veces pueden ser contradictorios. En los párrafos siguientes se hace un breve análisis de los datos correspondientes a este período y un intento de interpretación de los mismos.

\section{Cuadro $\mathrm{n}^{\mathrm{0}}$ 1. Valor añadido bruto (VAB) del sector industrial}

\begin{tabular}{|c|c|c|c|c|c|}
\hline \multirow{2}{*}{ Años } & \multicolumn{2}{|l|}{ Andalucía } & \multicolumn{2}{|l|}{ España } & \multirow{2}{*}{$\begin{array}{c}\text { \% industria andaluza } \\
\text { \% sobre industria española }\end{array}$} \\
\hline & $\begin{array}{c}\text { VAB en miles de millones } \\
\text { de ptas. de } 1981\end{array}$ & $\begin{array}{c}\pi \\
(1981=100)\end{array}$ & $\begin{array}{l}\text { VAB en miles de millones } \\
\text { de ptas. de } 1981\end{array}$ & $\begin{array}{c}\% \\
(1981=100)\end{array}$ & \\
\hline 1981 & 413.734 & 100,0 & 4.016 .835 & 100,0 & 10,3 \\
\hline 1983 & 424.149 & 102,5 & 4.241 .490 & 105,6 & 10,0 \\
\hline 1985 & 412.804 & 99,8 & 4.856 .518 & 120,9 & 8,5 \\
\hline 1987 & 453.449 & 109,6 & 4.534 .490 & 112,9 & 10,0 \\
\hline 1989 & 494.463 & 119,5 & 5.150 .656 & 128,2 & 9,6 \\
\hline 1991 & 481.714 & 116,4 & 4.769 .445 & 118,7 & 10,1 \\
\hline 1993 & 426.775 & 103,2 & 4.026 .179 & 100,2 & 10,6 \\
\hline
\end{tabular}

Fuente: BBV (varios años), Renta nacional de España y su distribución provincial, Bilbao, Ed. BBV.

Cuadro no 2. Índices de producción industrial

\begin{tabular}{|l|c|c|}
\hline Años & $\begin{array}{c}\text { Índice producción industrial } \\
\text { de Andalucía \%(Base 1990) }\end{array}$ & $\begin{array}{c}\text { Indice producción industrial de } \\
\text { España \%(Base 1990) }\end{array}$ \\
\hline 1990 & 100,0 & 99,6 \\
1991 & 96,6 & 98,8 \\
1992 & 87,1 & 96,0 \\
1993 & 67,4 & 91,6 \\
1994 & 83,3 & 97,8 \\
1995 & 84,0 & 103,6 \\
\hline
\end{tabular}

Fuente: Instituto de Estadística de Andalucía (1995).

Si cambiamos la base del cuadro no 2 para enlazar las dos series (cuadros $\mathrm{n}^{0} 1 \mathrm{y} \mathrm{n}^{0} 2$ ) obtendríamos para 1995 un índice de VAB industrial de Andalucía de 128,6 (base $100=1981$ ) y 113,3 para el ámbito español (también base $100=1981$ ). 
A partir de los datos anteriores, se pueden realizar algunos comentarios:

- Hasta el año 1993 los resultados de la industria han sido muy mediocres; lo mismo se podría decir con relación a la industria española.

- Durante el año 1994 y en menor medida en el 1995, la industria andaluza presenta unos buenos resultados que superan claramente los de la industria española en su conjunto.

- A partir del cuadro se pueden determinar cuatro etapas a lo largo del período: a) primera mitad de la década de los ochenta; comportamiento negativo, que coincide con los años de crisis de la economía mundial; b) segunda mitad de la década de los ochenta; expansión importante, más amplia que la que corresponde a la débil recuperación de la economía rnundial; c) primeros años de la década de los noventa; fuerte caída de la actividad industrial, correspondiente también a una etapa de recesión, cuyos efectos sobre la industria andaluza fueron especialmente virulentos; d) recuperación industrial, en el marco de una etapa ligeramente expansiva, a partir de 1994.

- La coyuntura económica nacional, y en su caso internacional, determinan claramente el comportamiento de la industria andaluza; los efectos sobre la comunidad autónoma andaluza se manifiestan, a veces, de forma retrasada, $\mathrm{y}$, otras veces, con un cierto adelanto sobre el ciclo económico español. Con frecuencia, la caída de la producción industrial andaluza es más profunda que la española, mientras que el crecimiento, de las etapas de auge, suele ser inferior.

- No se observa un desmantelamiento industrial, aunque sí aparece un cierto estancamiento.

- De todo lo anterior no se desprende necesariamente que la autonomía de Andalucía no haya tenido efectos positivos sobre la producción industrial o que la política industrial no haya alcanzado resultados positivos. Lo único que se puede afirmar es que la coyuntura económica española e internacional determinan la evolución industrial andaluza de una forma mucho más terminante que la posible influencia de las variables interiores. 
Hay que tener presenteque las fuentes de los dos cuadros utilizadas tienen un origen muy diverso por lo que las comparaciones pueden ser discutibles. Además, el poco tiempo durante el cual se ha estado elaborando el índice de producción industrial de Andalucía, habrá provocado, probablemente, algunos defectos e inexactitudes que se habrán ido corrigiendo durante los años más recientes. Por todo ello, hay que tratar con bastante cautela los resultados claramente positivos, de la industria andaluza en el último bienio.

\section{Comportamiento de los sectores de la industria anda- luza.}

En el cuadro $\mathrm{n}^{\mathrm{9}} 1$ se han tomado las cifras correspondientes a la industria sin incluir el subsector de la construcción; los resultados son más favorables si se añaden los datos de este subsector cuya importancia en términos absolutos así como su variación en el período, mucho más elevada que el de la industria propiamente dicha, permiten considerarlo como un subsector clave en la economía andaluza.

En el cuadro no 3 aparecen las variaciones del VAB de algunos subsectores:

Cuadro no 3. Variación del VAB de los subsectores industriales andaluces. Período 1983 a 1991.

\begin{tabular}{|l|c|}
\hline \multicolumn{1}{|c|}{ Subsectores } & \% Variación \\
\hline Construcción e ingeniería & 79,0 \\
Productos energéticos y agua & 63,0 \\
Papel y artes gráficas & 13,5 \\
Caucho y materiales plásticos & 13,0 \\
Material de transporte & 8,8 \\
Productos alimenticios, bebidas y tabaco & 6,5 \\
\hline
\end{tabular}

Fuente: BBV (varios años), Renta nacional de España y su distribución provincial, Bilbao, Ed. BBV. 
El resto de los subsectores presenta un estancamiento de la producción: madera y muebles, o un retroceso del VAB: minerales y metales, minerales y productos no metálicos, productos químicos, productos metálicos y maquinaria, textil y calzado.

También es importante subrayar que con relación a la industria española los únicos sectores que incrementan su cuota son, por orden de importancia, "los productos energéticos", el "caucho", la "construcción" y la industria agroalimentaria.

Los resultados son bien contundentes: sólo la construcción y la energía presentan un aumento importante, lo que significa un escaso crecimiento de la industria manufacturera, y una reducción de la ya escasa diversificación de la industria andaluza. Es importante señalar también que la construcción salva el pobre comportamiento de la economía andaluza y que los productos metálicos pierden relevancia; como datos positivos se puede indicar el crecimiento de la fabricación de material de transporte y de la industria agroalimentaria. Los subsectores "papel" y "caucho" aunque tengan un crecimiento moderado, tienen muy poca importancia, en términos relativos, dentro del sector industrial andaluz, por lo que su comportamiento positivo no tiene gran valor.

El cuadro $n^{0} 4$ recoge información de carácter sectorial, procedente de otra fuente distinta, y para un período algo más amplio que el anterior. 
Cuadro $\mathrm{n}^{\circ}$ 4. Variación del VAB de los sectores industriales andaluces. Período 1984 a 1995 (Noviembre)

\begin{tabular}{|l|r|}
\hline \multicolumn{1}{|c|}{ Subsectores } & \% Variación \\
\hline Construcción naval & 498 \\
Máquinas de oficinas y ordenadores & 419 \\
Maquinaria y material eléctrico & 233 \\
Otras industrias manufactureras & 99 \\
Instrumentos de precisión & 95 \\
Extracción de minerales no metálicos & 51 \\
Maquinaria y equipos mecánicos & 50 \\
Material electrónico & 46 \\
Productos alimenticios & 45 \\
Transformación de minerales no metálicos & 44 \\
Refino de petróleo & 38 \\
Otro material de transporte & 37 \\
Vehículos y piezas de repuesto & 36 \\
Energía eléctrica & 36 \\
Producción y primera transformación de metales & 33 \\
Industria química & -8 \\
Azúcar, bebidas y tabaco & -9 \\
Productos metálicos & -13 \\
Papel y artes gráficas & -18 \\
Calzado y vestido & -20 \\
Extracción de combustibles sólidos & -25 \\
Madera, corcho y muebles & -30 \\
Cuero & -39 \\
Textil & -45 \\
Caucho y materiales plásticos & -57 \\
Extracción y preparación de minerales metálicos & -70 \\
\hline
\end{tabular}

Fuente: Instituto de Estadística de Andalucia (1995).

A los datos del cuadro anterior, convendría añadir las cifras de variación de la producción industrial clasificada por el destino económico de los bienes, durante el período 1984-1995.

- Bienes de consumo $10 \%$

- Bienes de equipo $63 \%$

- Bienes intermedios $19 \%$ 
Un último dato a tener en cuenta es el del valor total de la nueva construcción, que en 1994 ascendió a un $72 \%$ del valor del mismo concepto correspondiente a 1990.

Los datos del período 1984-1995 muestran una evolución positiva de los sectores cuyos mercados presentan un mayor dinamismo a escala internacional y que, en general, tienen mayor capacidad de innovación. En este grupo de sectores de comportamiento positivo hay que incluir también la industria agroalimentaria, que como es sabido tiene un fuerte protagonismo en el sector secundario andaluz.

Por el contrario, los subsectores que han ocupado un lugar importante en la industria andaluza: textil, madera, etc., y que podríamos definir como sectores tradicionales, han tenido un fuerte quebrando en el período analizado.

Los buenos resultados de la producción de bienes de equipo y en menor medida de bienes intermedios, frente a un crecimiento muy pequeño de los bienes de consumo, representa un cambio profundo de las características tradicionales de la industria andaluza, en un sentido que estimamos acorde con un futuro más dinámico y estable del sector.

En cuanto al subsector de la "construcción" aunque sigue teniendo un lugar importante en la economía andaluza, una vez que se han agotado los efectos de las inversiones con motivo de la Expo-92, ha descendido su actividad en términos reales.

Estos cambios, positivos en líneas generales, de la industria andaluza se producen durante los años 1994 y 1995, nueva etapa expansiva de la economía española; la corta duración de este período impiden considerar que se ha producido un cambio estructural de la industria andaluza. Nos planteamos sin embargo una pregunta, que no es posible responder todavía: ¿los profundos cambios de la economía internacional han motivado este comportamiento de la industria andaluza, o bien ello es el resultado de la política industrial de la Junta de Andalucía? 


\section{La localización industrial.}

El cuadro $n^{0} 5$ recoge algunos datos relacionados con la industria desde un punto de vista geográfico.

Cuadro $\mathrm{n}^{\mathrm{0}}$. Comportamiento de la industria por provincias

\begin{tabular}{|l|c|c|c|}
\hline Provincias & $\begin{array}{c}\text { Industria sin construcción \% } \\
\text { sobre VAB regional. 1981 }\end{array}$ & $\begin{array}{c}\text { Industria sin construcción } \\
\text { Variación 1981-93(\%) }\end{array}$ & $\begin{array}{c}\text { Construcción Variación } \\
1981-93(\%)\end{array}$ \\
\hline Almería & 3,6 & 48 & 12 \\
Cádiz & 18,7 & 5 & 40 \\
Córdoba & 10,5 & 14 & 77 \\
Granada & 6,8 & 20 & 55 \\
Huelva & 13,3 & -29 & 109 \\
Jaén & 9,4 & 13 & 62 \\
Málaga & 11,2 & 9 & 67 \\
Sevilla & 26,5 & 2 & 120 \\
\hline
\end{tabular}

Fuente: $\mathrm{BBV}$ (varios años), Renta nacional de España y su distribución provincial, Ed. BBV, Bilbao.

Nota: Las variaciones se han calculado a partir de los datos del VAB a precios constantes.

Los datos son, de nuevo, muy elocuentes:

- La industria, en sentido estricto, ha tenido una trayectoria en el triángulo Sevilla- Cádiz-Huelva claramente insatisfactoria durante los trece años considerados. La cuarta provincia en producción industrial, Málaga, se ha comportado de forma nada más que medianamente aceptable. Las cuatro provincias restantes, las menos industriales, ha tenido crecimientos más positivos. Esta redistribución de la industria podría indicar una localización más diversificada del sector. Sin embargo, esta conclusión resulta menos positiva si subrayamos que se ha alcanzado más por un retroceso de las zonas tradicionalmente industriales andaluzas que por un crecimiento de las menos industrializadas.

- La construcción presenta resultados positivos en todas las provincias, compensando los malos resultados del resto de la industria. Las provincias que tienen variaciones más elevadas son Sevilla y Huelva y, en menor medida, Córdoba. Estos datos muestran una realidad diferente a la indicada en el 
párrafo anterior: reforzamiento de dos de las provincias con mayor porcentaje industrial, a lo cual no debe ser ajena la realización de infraestructuras y obras públicas en general.

\section{El empleo y otros aspectos del mercado de trabajo.}

En el periodo 1981 a 1991, la industria, sin incluir construcción, pasa de un empleo de 274.909 a 261.569 personas, un descenso por consiguiente de unos 13.500 empleados. Por el contrario en el subsector de la construcción hay un crecimiento del empleo de 160.700 a 211.569 , es decir unas 51.000 personas.

Los datos anteriores proceden de la publicación Renta Nacional de España y su distribución provincial. Otra fuente distinta: La encuesta de población activa, presenta los siguientes datos:

Cuadro n 6. Ocupados por sectores en Andalucía. Media Anual

\begin{tabular}{|c|cc|cc|}
\hline & \multicolumn{2}{|c|}{ Industria } & \multicolumn{2}{c|}{ Construcción } \\
\cline { 2 - 5 } Años & № de personas & $\begin{array}{c}\text { \% sobre ocupados en } \\
\text { España }\end{array}$ & N" de personas & $\begin{array}{c}\text { \% sobre ocupados en } \\
\text { España }\end{array}$ \\
\hline 1991 & 286.500 & 11,3 & 210.000 & 21,1 \\
1992 & 272.000 & 11,3 & 185.000 & 20,4 \\
1993 & 228.600 & 10,4 & 160.000 & 19,8 \\
1994 & 228.500 & 10,8 & 157.500 & 20,0 \\
1995 & 224.400 & 10,5 & 174.800 & 20,4 \\
\hline
\end{tabular}

Fuente: Instituto Nacional de Estadística (1996).

Por subsectores, los únicos que alcanzan un aumento de empleo son los de fabricación de productos metálicos, la elaboración de papel y los transformados del caucho, aparte de la actividad constructora ya citada. Los restantes sectores, o mantienen su empleo, en algún caso, o pierden empleados, lo más frecuente. Es necesario subrayar que un subsector como el de producción energética que tiene un fuerte crecimiento del $\mathrm{VAB}$, pierda empleo, y que otro 
subsector, el de "alimentación, bebidas y tabaco", cuya producción también aumenta en el período, mantenga el empleo, únicamente.

Desde el punto de vista del empleo, es más notable aún la importancia de la industria de la construcción, sin la cual la situación de paro se vería sustancialmente agravada.

En relación al empleo industrial en España, es interesante indicar que la industria andaluza ( $\sin$ la construcción) mantiene el porcentaje en los primeros años del período, mientras que este tanto por ciento desciende en el último trienio. La construcción en la región andaluza presenta un ligero aumento de su participación en el correspondiente sector nacional y un descenso, también ligero, en los últimos años. La economía andaluza, por tanto, tiene un problema respecto al empleo industrial, que es común al de la economía española. Se deduce también que el crecimiento de la producción no contribuye a solucionar dicho problema, sino que va unido a pérdidas de empleo, ya que las nuevas inversiones son intensivas en capital y ahorradoras de empleo.

Otro aspecto a considerar en relación con el empleo es el del coste laboral. Éste que es inferior a la media nacional, se acerca al valor medio en los primeros años del período autonómico, hasta 1983, volviendo luego a separarse del promedio, a causa de un menor nivel del coste salarial andaluz que se sitúa en un $90 \%$ del estatal, al final de la década.

En cuanto a la productividad por trabajador, se sitúa en 1991 por encima del valor al principio del período estudiado, con un incremento de un $22 \%$ aproximadamente. Sin embargo en términos relativos, la productividad andaluza que superaba a la media española en 1980, pasa a ser similar a la misma en 1990. Estos datos podrían hacer pensar en una menor competitividad de la industria andaluza; sin embargo el menor nivel del coste salarial, y su comportamiento durante este período, compensa esta diferencia, permitiendo una ventaja competitiva del sector respecto a la industria nacional, que se mantienen a lo largo de toda la etapa autonómica. 


\section{Las principales causas que han determinado el comportamiento de la industria andaluza.}

Las distintas etapas por las que ha pasado la industria andaluza, durante el período autonómico, muestran una gran sensibilidad a las distintas fases del ciclo económico, que en el caso andaluz determina, normalmente, variaciones más amplias que en otros espacios económicos. Ello parece lógico si consideramos el importante grado de apertura de la economía regional, unido a defectos estructurales de su industria, todo lo cual determina una especial dependencia de la industria regional respecto a las variaciones de la economía mundial.

La proporción del VAB regional sobre el nacional, que se ha mantenido casi invariable durante estos años, está determinada por la estrecha relación de Andalucía con el resto de España, por lo que existen causas comunes que orientan en el mismo sentido los respectivos sectores industriales. No se puede defender, por lo tanto, una evolución aislada de la industria andaluza.

A lo largo de estos años, la necesidad de competir en los mercados nacional e internacional ha obligado a la aplicación de nuevas tecnologías en este sector que han conducido a una moderada modernización del mismo. Estos cambios unidos al comportamiento de los salarios por debajo del promedio nacional, han permitido una mejora de la competitividad de los productos industriales de Andalucía.

Por otra parte, algunas industrias con capital del resto de España o del exterior han abandonado sus instalaciones en Andalucía. Esto se ha producido, en los primeros años de la etapa autonómica, en los subsectores de la industria transformadora no agraria, principalmente, lo que ha determinado una mayor concentración de la producción industrial agroalimentaria.

Sin embargo, la evolución positiva del último bienio que ha ido unida a una sectorización de mejor futuro, puede ser el resultado de la nueva división internacional del trabajo en el sector industrial que, quizás, ha sido favorable para la comunidad autónoma; los cambios, muy negativos para la industria andaluza, que se produjeron en los años setenta y ochenta, aconsejan no adoptar una postura demasiado optimista antes de conocer la trayectoria industrial de los próximos años.

La industria constructora ha obtenido los mejores resultados del período, lo que ha sido causado por una elevada actividad de obras públicas y de 
La industria constructora ha obtenido los mejores resultados del período, lo que ha sido causado por una elevada actividad de obras públicas y de infraestructura y por una demanda creciente de vivienda en las etapas de auge, fenómeno este último, la demanda de vivienda, que parece haberse frenado en los últimos meses.

El incremento de las exportaciones al extranjero, puede ser debida a la mejora de la competitividad de la industria y a la modernización del algunos productos; los datos sobre las mejoras técnicas aplicadas en la industria mundial hacen pensar, sin embargo, que en este buen comportamiento de las exportaciones ha tenido un papel relevante la adhesión a la Comunidad económica europea.

\section{La política pública regional en relación con la indus- tria en el contexto de la planificación económica.}

Desde la constitución de la comunidad autónoma andaluza, e incluso en la etapa preautonómica, se ha dado una especial importancia a la realización de una política planificadora, meramente indicativa de la economía andaluza.

En todos los planes aprobados durante estos años, aparece el sector industrial en un lugar destacado en el orden de prioridad de los sectores económicos. Ha habido, sin embargo, pequeños cambios en relación a la relevancia asignada al sector. En cualquier caso, el análisis del tratamiento de la industria en los distintos planes es muy importante para conocer la política industrial realizada por la Junta de Andalucía.

Los documentos que vamos a utilizar son los siguientes:

- Plan económico para Andalucía. 1984-86 (PEA)

- Programa andaluza de desarrollo económico. 1987-1990 (PADE)

- Plan andaluz de desarrollo económico. 1991-1994 (PADE II)

- Programa industrial para Andalucía (1994-1997) (PIA)

Una primera observación que se desprende de la lectura de estos programas es la heterogeneidad formal de los mismos. En algunos de ellos se establecen objetivos sectoriales, en uno de ellos los objetivos tienen un carácter general. Varían los nombres de los distintos elementos de la planificación; en concreto el concepto de programa tiene un significado diferente en los distintos planes. Todo esto tiene efectos negativos importantes sobre la eficacia 
de la política planificadora. Esta heterogeneidad es tanto más sorprendente, cuanto que durante el período autonómico el partido gobernante ha sido siempre el mismo. ¿Qué hubiera pasado si se hubiera producido una alternancia en el poder?

Es importante subrayar que el último documento tiene un carácter sectorial, por lo que la comparación con los otros puede ser discutible. Su contenido, limitado al sector industrial, le da, sin embargo, una especial importancia con relación a este trabajo.

\subsection{Los objetivos}

Analizamos en primer lugar, los objetivos de los diferentes planes en relación con el sector industrial. Los datos se recogen en el cuadro $n^{0} 7$.

\section{Cuadro $n^{\circ} 7$. Objetivos en relación con el sector industrial}

\begin{tabular}{|c|c|c|c|}
\hline $\begin{array}{l}\text { Plan económico } \\
\text { Andalucía 84-86 }\end{array}$ & $\begin{array}{c}\text { Programa andaluz } \\
\text { de desarrollo económico } 87-90\end{array}$ & $\begin{array}{l}\text { Plan andaluz de desarrollo } \\
\text { económico para } 91-94\end{array}$ & $\begin{array}{l}\text { Programa industrial para } \\
\text { Andalucia } 94-97\end{array}$ \\
\hline $\begin{array}{l}\text { - Mayor participa- } \\
\text { ción en la estructura } \\
\text { económica regional. } \\
\text { - Ajuste estructura } \\
\text { industrial a nuevas } \\
\text { condiciones económicas. } \\
\text { - Lograr una estruc- } \\
\text { tura más articulada. }\end{array}$ & $\begin{array}{l}\text { - Creación de empleo } \\
\text { estable. } \\
\text { - Aumento de la renta } \\
\text { y retención de ésta en } \\
\text { Andalucia. } \\
\text { - Mejor utilización del } \\
\text { potencial endógeno. } \\
\text { - Creación de empre- } \\
\text { sas de tamaño medio. } \\
\text { - Importación de I+D. } \\
\text { - Fomento de activida- } \\
\text { des de fuerte implanta- } \\
\text { ción regional y con futuro. } \\
\text { - Prioridad a las } \\
\text { industrias emergentes de } \\
\text { carácter innovador. } \\
\text { - Potenciación y diver- } \\
\text { sificación de la estructura } \\
\text { industrial: }\end{array}$ & $\begin{array}{l}\text { al Estrategia de racio- } \\
\text { nalización de los sectores } \\
\text { de peso en Andalucia. } \\
\text { b) Estrategia de desa- } \\
\text { rollo exógeno de sectores } \\
\text { en expansión y en los que } \\
\text { Andalucia cuenta con } \\
\text { pocas ventajas competiti- } \\
\text { vas. } \\
\text { c) Acciones puntuales } \\
\text { de proyectos viables de } \\
\text { sectores con perspectivas } \\
\text { de bajo crecimiento. }\end{array}$ & $\begin{array}{l}\text { - Consolidación, } \\
\text { expansión y diversifica- } \\
\text { ción de la industria. } \\
\text { - Generación de } \\
\text { empleo estable. } \\
\text { - Adecuada distribu- } \\
\text { ción en el territorio. } \\
\text { - Mejora de la capaci- } \\
\text { dad competitiva de la } \\
\text { industria andaluza. } \\
\text { - Desarrollo y diversifi- } \\
\text { cación de subsectores con } \\
\text { perspectivas de creci- } \\
\text { miento y carácter dinámi- } \\
\text { co (desarrollo exógeno de } \\
\text { forma no selectiva). }\end{array}$ \\
\hline
\end{tabular}

Fuente: Junta de Andalucía (1985), (1988), (1991) y (1994). 
Hay que tener en cueñta que el programa 87-90 (PADE) contiene únicamente objetivos generales, no incluyendo por lo tanto objetivos específicos para el sector industrial; esto hace complicada la comparación con los otros programas; no obstante, la importancia que el PADE da a la industria en el contexto de la economía andaluza, permite considerar que estos objetivos son aplicables, en líneas generales, al sector industrial, no planteando grandes problemas a la comparación interprogramas.

Los principales objetivos que aparecen en el cuadro $\mathrm{n}^{0} 7, \mathrm{y}$ siguiendo un orden cronológico, son los siguientes:

- Aumento de la importancia del sector industrial; es decir, industrialización de Andalucía.

- Incremento del empleo industrial, de carácter estable.

- Potenciación de las actividades industriales endógenas.

- Aumento del tamaño de la empresa, aunque teniendo como objetivo un tamaño medio.

- Gran importancia del desarrollo tecnológico.

- Prioridad de las industrias innovadoras.

- Diversificación de subsectores o ramos industriales.

- Desarrollo industrial exógeno de ciertas ramas industriales.

- Redistribución territorial.

- Mejora de la competitividad.

Se observa una cierta coherencia en relación a los objetivos, si bien cada uno de los documentos tiene algún aspecto que los singulariza. Así, por ejemplo: a) el PEA insiste en la problemática creada por la crisis industrial; b) el $\mathrm{PADE}$ subraya el potencial endógeno en relación a la estrategia industrial; c) el PADE II utiliza el concepto de desarrollo exógeno y la idea de racionalización; y d) el PIA pone el énfasis en la mejora de las condiciones del sector y de la competitividad.

Estos cambios de orientación, que serían razonables en un período de tiempo más largo, no son aceptables en un horizonte de catorce años. Estimamos que una variación de objetivos, o de alguna parte de los objetivos, cada tres o cuatro años, se puede, considerar como un cambio de lá política industrial que puede afectar profundamente a la eficacia de la misma. 


\subsection{Prioridades de los subsectores}

El cuadro $n^{2} 8$ contiene los diferentes subsectores industriales que se han destacado como prioritarios en cada uno de los programas

\section{Cuadro $n^{\circ} 8$. Subsectores o ramas industriales prioritarias}

\begin{tabular}{|c|c|c|c|}
\hline $\begin{array}{l}\text { Plan económico } \\
\text { Andalucía } 84-86\end{array}$ & $\begin{array}{c}\text { Programa andaluz } \\
\text { de desarrollo económico } 87-90\end{array}$ & $\begin{array}{l}\text { Plan andaluz de desarrollo } \\
\text { económico para } 91-94\end{array}$ & $\begin{array}{c}\text { Programa industrial para } \\
\text { Andalucia } 94-97\end{array}$ \\
\hline $\begin{array}{l}\text { - Industrias de trans- } \\
\text { formación agraria. } \\
\text { - Industrias auxiliares } \\
\text { de la agricultura. } \\
\text { - Aprovechamniento de } \\
\text { materias primas. } \\
\text { - Sectores de alto cre- } \\
\text { cimiento: } \\
\text {-electrónico } \\
\text {-telecomunicaciones } \\
\text {-bioindustria } \\
\text { - Energia }\end{array}$ & $\begin{array}{l}\text { - Complejo agroindus. } \\
\text { trial lindustria agroali- } \\
\text { mentaria, quimica, } \\
\text { piensos y abonos, semi- } \\
\text { llas y productos, } \\
\text { maquinaria agricola, } \\
\text { sistemas de riego, etc.). } \\
\text { - Energia solar. } \\
\text { - Microelectronica. } \\
\text { - Tecnologias de la infor- } \\
\text { macion. } \\
\text { - Mineria. } \\
\text { - Energía }\end{array}$ & $\begin{array}{l}\text { - Industria agroalimen- } \\
\text { taria. } \\
\text { - Material electrico y } \\
\text { electrinico. } \\
\text { - Material de transporte. } \\
\text { - Maquinaria y equipo. } \\
\text { - Caucho y materias } \\
\text { plásticas. } \\
\text { - Productos minerales nu } \\
\text { metálicos. } \\
\text { - Textil, calzado y cueru. } \\
\text { - Madera, corcho y mue- } \\
\text { bles. } \\
\text { - Artes gráficas y edi- } \\
\text { ción. } \\
\text { - Energia. } \\
\text { - Mineria. }\end{array}$ & $\begin{array}{l}\text { - Industria agrualimen- } \\
\text { taria. } \\
\text { - Material electrónico e } \\
\text { informatico. } \\
\text { - Material de transporte: } \\
\text {-Automoción } \\
\text {-Aeronautico } \\
\text { - Naval } \\
\text { - Quimica. } \\
\text { - Medioambiental. }\end{array}$ \\
\hline
\end{tabular}

Fuente: Junta de Andalucia (1985). ( 1988), ( 1991) y (1994).

De nuevo nos encontramos con una gran diversidad de denominaciones que hace difícil comparar las distintas columnas. Otro aspecto que da lugar a una cierta heterogeneidad de los datos es que en alguno de los documentos se da una relación de prioridades abierta, de forma que es difícil determinar que sectores tendrían o no tendrían cabida en estas relaciones de prioridades.

Del cuadro se deduce que existen diversos sectores que aparecen más de una vez en los distintos documentos:

- Industria agraria

- Industria auxiliar de la agricultura

- Industria eléctrica y electrónica

- Industria de telecomunicaciones 
- Industria energética

- Industria minera

- Industria de material de transporte

- Industria de maquinaria y equipo

- Industria química

- Industria medioambiental y bioindustria.

Otras ramas que no se citan, de forma expresa, nada más que una sola vez, son las siguientes:

- Caucho y materias plásticas

- Textil, calzado y cuero

- Madera, corcho y muebles

- Artes gráficas y edición.

Los datos anteriores nos muestran que la prioridad de sectores industriales se mantiene en su mayor parte a lo largo del período analizado, lo cual puede dar una gran estabilidad a la política industrial.

Es importante subrayar que ciertos planteamientos recientes, o menos recientes, relacionados con los aspectos medioambientales, con el desarrollo sostenible, etc., se han tenido en cuenta en la política industrial, aunque desconocemos los resultados prácticos de estos enfoques.

Se producen, sin embargo, ciertos cambios, algunos de ellos, quizás, solo aparentes, que pueden afectar negativamente a esta política. Por ejemplo, en el programa industrial no aparecen la energía, ni la minería ¿es que han dejado de tener carácter prioritario? De igual forma, la industria de material de transporte, que tanta importancia ha tenido en algunas zonas andaluzas, no aparecen ni en el PEA, ni en el PADE. Otros sectores tradicionales de la industria andaluza, tales como textil, madera, etc., que han sufrido graves quebrantos en las dos últimas décadas, solo aparecen en el PADE II. Todos estos cambios nos indican variaciones, difícilmente comprensibles, si se pretende dar estabilidad a la política industrial.

\subsection{Medios o instrumentos}

Los datos relacionados con los medios o instrumentos de la política industrial se incluyen en el cuadro $\mathrm{n}^{2} 9$. 
Aquí aparecen, de nuevo, problemas terminológicos y conceptuales, ya que la separación entre los objetivos o fines de la política y los medios o instrumentos no siempre es rigurosa. Algunos de los datos de este cuadro deberían trasladarse al cuadro $\mathrm{n}^{0} 7$; sin embargo, los planes los denominan medios o instrumentos, por lo que hemos decidido mantenerlos en este apartado

\section{Cuadro $\mathrm{n}^{\circ}$ 9. Medios o instrumentos de la política industrial}

\begin{tabular}{|c|c|c|c|}
\hline $\begin{array}{l}\text { Plan económico } \\
\text { Andalucia } 84-86\end{array}$ & $\begin{array}{c}\text { Programa andaluz } \\
\text { de desarrollo económico } 87-90\end{array}$ & $\begin{array}{c}\text { Plan andaluz de desarrollo } \\
\text { económico para } 91-94\end{array}$ & $\begin{array}{c}\text { Programa industrial para } \\
\text { Andalucia } 94-97\end{array}$ \\
\hline $\begin{array}{l}\text { - Mejora de la infraes- } \\
\text { tructura industrial: } \\
\text { - Acciones de reindustria- } \\
\text { lización: } \\
\text { - Realización de planes de } \\
\text { fomento industrial. } \\
\text { - Reconversión y reestruc- } \\
\text { turación industrial. } \\
\text { - Apoyo a la innovación y } \\
\text { desarrollo tecnológico. } \\
\text { - Mejora de los servicios } \\
\text { territoriales y de inspec- } \\
\text { ción. } \\
\text { - Realizacion de estudios } \\
\text { y estadisticas industria- } \\
\text { les. }\end{array}$ & $\begin{array}{l}\text { - Seleccion. adaptación y } \\
\text { aplicación de tecnologia } \\
\text { exterior. } \\
\text { - Movilizacion de los recur- } \\
\text { sos financieros internos y } \\
\text { atracción de recursos } \\
\text { externos. } \\
\text { - Formación de capital } \\
\text { humano. } \\
\text { - Mejora de la competitivi- } \\
\text { dad. } \\
\text { - Acceso a los mercados } \\
\text { nacional e internacional. } \\
\text { - Prestación de servicios por } \\
\text { la administracion regional. } \\
\text { - Mejora de la integracion } \\
\text { territorial. }\end{array}$ & $\begin{array}{l}\text { - Fomento de la concentra- } \\
\text { ción de empresas. } \\
\text { - Creación de marcas. } \\
\text { - Fomento de cooperativas. } \\
\text { - Limitacion de la entrada } \\
\text { de capitales extranjeros en } \\
\text { sectores tradicionalmente } \\
\text { andaluces. } \\
\text { - Articulacion de iniciarivas } \\
\text { exógenas con el tejido pro- } \\
\text { ductivo andaluz. } \\
\text { - Seguimiento de iniciativas } \\
\text { exogenas incentivadas. } \\
\text { - Consolidacion de los } \\
\text { nucleos industriales emer- } \\
\text { gentes. } \\
\text { - Apoyo al ahorro y diversi- } \\
\text { ficación energetica. } \\
\text { - Investigacion geologica. } \\
\text { - Plan de seguridad minera. } \\
\text { - Potenciación de las SGR }\end{array}$ & $\begin{array}{l}\text { - Modernización empresa- } \\
\text { rial. } \\
\text { - Modernizacion tecnologica. } \\
\text { - Suelo industrial } \\
\text { - Fomento de la financia- } \\
\text { cion. } \\
\text { - Ordenacion y control. } \\
\text { - Formacion empresarial. } \\
\text { - Creacion y mejora de infra- } \\
\text { estructura energetica. } \\
\text { - Infraestructuras de trans- } \\
\text { portes y comunicaciones. }\end{array}$ \\
\hline
\end{tabular}

Fuente: Junta de Andalucía (1985), (1988), (1991) y (1994).

A partir del cuadro $\mathrm{n}^{\circ} 9$ y de los programas relacionados con el sector industrial que se incluyen en cada uno de los documentos que estamos analizando, se puede realizar la siguiente tipología de instrumentos de la política industrial.

- Creación de infraestructuras.

- De transporte y comunicaciones

- Energética

- Industrial: suelo industrial y naves modulares. 
En algunos casos, la àcción pública se concreta en la subvención a la iniciativa privada que tendrá la titularidad de las instalaciones.

- Subvenciones y apoyos públicos.

Las actividades subvencionables corresponden a las siguientes:

- Innovación y desarrollo tecnológico.

- Formación de capital humano.

- Acceso a mercados interiores y exteriores.

- Marcas y diseño.

- Modernización empresarial

- Concentración empresarial.

- Financiación de iniciativas de origen regional.

- Participación en empresas públicas de servicios.

- Ordenación de la entrada de capitales exteriores.

- Participación en el capital de empresas.

- A través de una sociedad de capital riesgo.

- Directamente (caso de las sociedades de garantía recíproca).

La instrumentación de la política industrial es bastante coherente y establece claramente el terreno que corresponde a la iniciativa pública -creación de infraestructuras y fomento del sector privado-, y el que corresponde a la iniciativa privada -las actividades directamente productivas-. Lógicamente las empresas públicas tienen como finalidad proporcionar servicios a las empresas privadas que les permitan competir en los mercados; no estando previsto, en consecuencia, la creación de empresas públicas que proporcionen bienes o servicios comercializables en los mercados.

Este reparto de competencias se ha ido concretando conforme se perfeccionaba la planificación económica. Por ejemplo, en el PEA no estaba claramente definido el papel de la empresa pública, y es ya en el PADE en el que queda establecido de forma precisa este modelo. Las alternativas, que pudiéramos denominar ideológicas, del gobierno andaluz pueden explicaŕ también, en parte, este proceso de maduración.

Con relación a los subprogramas relacionados con el sector industrial, convendría realizar un par de comentarios: 
$\left.1^{\mathbf{9}}\right)$ La política industrial no se agota con estos subprogramas, ya que hay otros, sobre todo los relacionados con las inversiones públicas, que tienen una importante influencia sobre el sector. La dificultad de diferenciar, en algunos casos, en qué parte responden a un objetivo de política industrial y en qué parte a otros objetivos, impide incluir, a veces, estos otros subprogramas.

$\left.2^{2}\right)$ La forma de establecer los subprogramas fue, en general, bastante simple: se solicitaban a los distintos organismos de la Junta, los programas o subprogramas que querían proponer a partir de unos documentos previos sobre objetivos, estrategias, etc. Los organismos enviaban los programas que habían elaborado anteriormente, o redactaban alguno nuevo. En principio, este método parece razonable ya que las líneas de política, que ya estaban en marcha, del gobierno andaluz deberían formar parte de la planificación. Pero lo que no parece tan razonable es que la selección de acciones por parte del organismo responsable de la planificación haya sido casi inexistente. De esta forma el carácter planificador de la política quedaba casi vacío de contenido.

\section{Otras acciones de política industrial.}

Otra actuación importante de la política industrial andaluza se realiza a través del Instituto de Fomento de Andalucía, cuyo antecedente el Instituto de Promoción Industrial Andaluz (IPIA), estaba especializado concretamente en este sector. El IFA mediante ayudas financieras, convenios con entidades financieras, participaciones en capital y programas sectoriales realiza una importante actividad de promoción industrial.

La modernización técnica se ha instrumentado, aparte de por otros medios, mediante los parques tecnológicos: el parque tecnológico de Andalucía en Málaga y Cartuja 93 en Sevilla. Estas iniciativas pueden ocupar un lugar sustancial en la política del sector, si bien aún no han llegado a madurar, por lo que sus actuaciones no han tenido todavía efectos reseñables.

También puede ser interesante determinar cuáles han sido las acciones de política industrial de la Junta de Andalucía durante la última legislatura ${ }^{4}$. Una revisión de las decisiones del ejecutivo regional nos muestra que las

4. Como es sabido, el gobierno andaluz ha estado en minoría desde Junio de 1994, lo que ha frenado decisiones importantes, hasta el punto de provocar un adelanto considerable de las elecciones, que se celebraron el 3 de Marzo de 1996. 
acciones de política industrial han tenido únicamente un carácter de trámite burocrático; especialmente, la numerosas aprobaciones de ayudas y subvenciones -entre las que destacamos las relacionadas con la modernización tecnológica- y los convenios con empresas públicas y privadas y otros entes.

Las únicas disposiciones de un mayor calado político son las que relacionamos a continuación.

- Aprobación de los programas:

A) de ayudas para la promoción y desarrollo del sector industrial

B) de ayudas para la modernización tecnológica y empresarial del sector industrial.

- Declaración de dos nuevas zonas de acción especial.

- Aprobación del II Plan andaluz de investigación.

- Aprobación del convenio con entidades crediticias para la financiación de pequeñas y medianas empresas.

- Ratificación de acuerdos del IFA en relación con la participación en el capital de empresas.

Asimismo, y en un contexto más amplio, convendría recordar que el PADE II finalizó en 1994, no habiéndose aprobado hasta la fecha un nuevo plan de desarrollo.

La escasa importancia de las decisiones sobre el sector industrial durante más de un año y medio nos permiten afirmar que este ha sido un período de tiempo perdido para la política industrial. Quizás, la única excepción haya sido el IFA, que dada la autonomía de que gozan sus decisiones, ha podido mantener su línea de actuación.

\section{Conclusiones.}

El sector industrial de la comunidad autónoma andaluza se ha desarrollado escasamente desde el inicio de la autonomía regional. Se podría decir que ha existido un casi estancamiento de la actividad productiva. Sin embargo, sería inadecuado mantener que se ha producido un desmantelamiento industrial durante esto años. Es cierto, que la nueva distribución de la actividad económica, en un contexto cada vez más globalizado, ha llevado a la desa- 
parición de empresas y, a veces, casi de sectores o subsectores, lo cual ha incrementado la concentración de la industria, sobre todo en el sector agroalimentario.

Convendría matizar el párrafo anterior en el sentido de que durante los años 94 y 95 los resultados son mucho más favorables, lo que podría, quizás, indicar un cambio de tendencia e incluso una nueva estructura industrial andaluza.

El problema es más grave desde el punto de vista del empleo, ya que el modesto desarrollo industrial ha sido incapaz de reducir, ni siquiera mantener, el paro de origen industrial. Esto, no obstante, es un problema generalizado a otros espacios económicos, por lo que parece discutible que los agentes económicos andaluces sean capaces de resolverlo por sí mismos.

Desde el punto de vista geográfico se han producido pequeños cambios que no han afectado de forma sustancial a los desequilibrios económicos e industriales, en concreto, que existen en Andalucia.

Si comparamos la ambiciosa política industrial de la Junta con el insatisfactorio comportamiento de la industria regional, tenemos que subrayar que los defectos de esta política ya indicados, la falta de medios para llevarla adelante y la presión de la coyuntura económica han impedido que se alcanzaran los objetivos establecidos.

Más en detalle, podemos afirmar que el tratamiento del sector industrial en los programas económicos de la Junta ha sido correcto desde el punto de vista de los objetivos, prioridades y medios o instrumentos. Existen diferencias apreciables, sin embargo, entre los distintos planes; así, si pretendemos expresar cada uno de ellos con una definición concisa, podríamos dar las siguientes: a) el PEA corresponde a la expresión "reconversión industrial"; b) el PADE, a "potencial endógeno"; c) el PADE II, a la "racionalización"; y d) el PIA a la "competitividad".

Los cambios, no muy profundos, en los aspectos indicados (objetivos, sectores y medios) deben haber reducido la eficacia de esta política. Así por ejemplo, algunos aspectos en los que se han producido cambios importantes de la política industrial son los siguientes: a) el papel de la empresa pública en el desarrollo industrial; b) el carácter endógeno o exógeno de las nuevas industrias; c) la prioridad de los sectores tradicionales de la industria andaluza o la 
de los sectores innovadores; d) la forma de incorporar las nuevas tecnologías; etc. En general, los defectos de la política planificadora del ejecutivo andaluz: escasa difusión de los programas, inadecuado control de su ejecución, desinterés de los agentes respecto a los mismos, etc. ${ }^{5}$, han contribuido a que los efectos de la política de planificación se hayan ido diluyendo gradualmente. Tampoco hay que olvidar que el "parón" que ha sufrido la política industrial durante la última legislatura ha frenado los posibles efectos de esta política. Dado que en estos dos años, 94 y 95, se han producido, precisamente, mejoras importantes de los resultados de la industria andaluza, se podría concluir, de forma apresurada, que la política industrial no ha tenido ningún efecto sobre el sector. Creemos, sin embargo, que el carácter de política a largo plazo de la industria, exige un horizonte temporal bastante largo para juzgar de su eficacia; todavía nos parece prematuro un juicio en este sentido.

Por otra parte, los efectos mas importantes sobre la actividad industrial han procedió de la política, no sólo de la Junta, de infraestructuras y de obras públicas. Estas acciones han estado motivadas, en parte, por la organización de la exposición del 92 , lo que unido a las características de la industria de la construcción, hace ver el carácter posiblemente efímero de esta política.

En resumen, ¿ha sido beneficiosa la política andaluza para el sector industrial de la comunidad autónoma? La respuesta podría ser negativa, puesto que no se han logrado los objetivos que se establecieron para la política industrial. Pero no hay que olvidar que la industria española, en general, y la andaluza, se encuentran ante la doble competencia de unos países desarrollados con niveles de salarios elevados, pero al mismo tiempo con una tecnología de punta y de unos países en desarrollo con niveles técnicos bajos, pero con unos salarios medios reducidos que les permiten competir frente a países más desarrollados. La situación intermedia entre estos dos bloques hace extraordinariamente difícil el desarrollo industrial andaluz.

La política de la Junta no puede pretender lograr, en solitario, un cambio radical de la situación del sector. Las decisiones de los grandes conglomerados industriales, cuyos centros de poder no se encuentran localizados, ciertamente, en Andalucía, pueden hacer fracasar una política industrial por adecuada que sea. De forma global pensamos que la Junta ha diseñado una

5. A la publicación de cada uno de los programas citados, se le dió una difusión amplia, de forma que pudo ser conocido po rlos distintos agentes económico-sociales; sin embargo, los informes periódicos sobre su ejecución han sido desconocidos para el ciudadano andaluz, y, con frecuencia, han quedado "olvidados" en un cajón. 
do recursos, no sólo financieros -pensamos, por ejemplo, en las competencias a este respecto- para llevar adelante esta política. Además, la realidad ha demostrado que para que dicha política tenga efectos positivos es necesario aplicarla pacientemente durante un período de tiempo más largo que los 15 años de la etapa autonómica. Asimismo, se trata de un problema de tal envergadura que exige la actuación y la coordinación de los otros niveles de la administración: la central y la de la Unión Europea.

Por otra parte, para lograr este objetivo es necesario que la iniciativa privada de Andalucía, aporte capitales y gestión y al mismo tiempo que se produzcan inversiones procedentes del exterior, que dentro de un marco que limite las operaciones especulativas, aporten recursos, nuevos procedimientos técnicos y organización adecuada.

En conclusión, la política sólo puede poner las condiciones que hagan posible el desarrollo, pero la creación de nuevas industrias exige la participación de la iniciativa privada. No existe, por tanto, ninguna fórmula que asegure el desarrollo industrial; sólo medidas adecuadas que lo faciliten. 
ANEXO. Programas y subprogramas relacionados con el sector industrial

\section{Plan Económico para Andalucía 1984-86}

Programa 7. Actuación de promoción y reestructuración de la industria y la minería Energía.

Mejora de las condiciones de infraestructura:

- Suelo industrial (GESTORES)

- Construcción de naves industriales

Participación en capital y préstamos directos por SOPREA

Plan de fomento a la artesanía andaluza

Fomento a la industrialización de productos agrarios

Investigación y desarrollo tecnológico:

- Apoyo a la incorporación de nuevas tecnologías

- Apoyo proyectos de I + D

- Investigación básica y aplicada

Mejora de los servicios territoriales y de los servicios de inspección:

- Ampliación de estaciones de ITV

- Mecanización administración de estaciones de ITV

- Estudios y estadísticas

Fomento a la minería

Fomento al ahorro energético y aprovechamiento de nuevas ind. residuos sólidos

\section{Programa andaluz de desarrollo económico 1987-1990}

Programas de industria

Investigación de Minerales Piríticos vía Pirrotita Apoyo a Proyectos de Aprovechamiento de Recursos Mineros Investigación Geológica y de Recursos Minerales Estudio sobre la Infraestructura Geológica-Minera

Diversificación Energética

Electrificación rural

Fomento del Aprovechamiento de Energías Renovables Ahorro Energético 
Ayudas a Empresas en Crisis del Sector Industrial

Desarrollo de la Calidad y Servicios Industriales

Profundización y Difusión del conocimiento del Sector Industrial

Construcción y Adaptación de Naves Industriales

Gestión de la Inspección Técnica de Vehículos

Creación de Zonas de Acción Especial

\section{Plan andaluz de desarrollo económico 1991-1994}

Apoyo a la industria agroalimentaria

Ordenación y promoción industrial:

- Ordenación industrial

- Control industrial

- Infraestructura industrial y apoyos sectoriales

- Desarrollo tecnológico y mejora de la productividad

- Fomento de la calidad y servicios industriales.

- Documentación, formación de recursos humanos e información industrial

Aprovechamiento de recursos mineros

Investigación geológica de los recursos mineros

Promoción y desarrollo minero

Corrección del impacto ambiental en extracciones mineras

Infraestructura Geológico-Minera de Andalucía

Plan de Seguridad Minera

Promoción tejido industrial:

- Apoyo a proyectos de interés regional

- Planes de actuación sectoriales

- Promoción exterior

Mejora de la estructura empresarial:

- Información, asistencia técnica y asesoramiento empresarial.

- Formación empresarial

- Financiación PYMES

Incentivos económicos a la creación de empresas:

- Ayudas complementarias a proyectos empresariales beneficiados por la Ley de Incentivos Regionales

- Actuaciones territoriales

- Parque Tecnológico de Andalucía

Fomento energético:

- Fomento de la utilización de las energías renovables

- Apoyo a la extensión de la infraestructura gasista 
- Apoyo al ahorro y diversificación energética

- Promoción de la electrificación del medio rural

Mejora de la competitividad:

- Innovación y calidad industrial

\section{Programa industrial para Andalucía 1994- 1997}

Desarrollo de Subsectores Preferentes

Fomento de otras Actividades Industriales

Mejora de la Competitividad y Modernización

Modernización Tecnológica

Infraestructura Industrial

Ordenación y Control Industrial

Apoyo Financiero a las PYMES

Infraestructuras de Apoyo

Formación

Promoción de la Economía Social

Promoción Comercial

Formación Profesional Reglada

Formación Universitaria Tecnológica

Plan Andaluz de Investigación

Infraestructuras de comunicaciones terrestres

Infraestructuras portuarias 


\section{Bibliografía}

Alcaide Castro, M. (1992), "La industria andaluza: el comportamiento espacial y sectorial de la inversión durante el período 1985-1990", en Revista de Estudios Regionales, núm. 32, pp. 15-42.

Castells, M. y Hall, P. (Dir.) (1992), Andalucia: Innovación Tecnológica y Desarrollo Económico, Madrid, Ed. Espasa Calpe.

CuAdradó Roura, J. R. (1987), Los cambios tecnológicos y el futuro económico de Andalucía, Sevilla, Ed. Instituto de Desarrollo Regional.

Delgado, M. y Román, C. (Eds.) (1995), Ocho análisis de la economía andalu$z a$, Sevilla, Ed. Instituto de Desarrollo Regional.

IFA (1991), El sector industrial en Andalucia, Sevilla, Ed. Servicio de Estudios y Publicaciones.

Junta De Andalucia (1985), Plan económico para Andalucía 1984-1986, Sevilla, Ed. Oficina de Planificación de la Consejería de Economía.

- (1988), Programa andaluz de desarrollo económico 1987-1990, Sevilla, Ed. Servicio de Publicaciones de la Consejería de Economía y Fomento.

- (1991), Plan andaluz de desarrollo económico 1991-1994, Sevilla, Ed. Servicio de Asesoría Técnica y Publicaciones de la Consejeria de Economía y Hacienda.

- (1994), Programa industrial para Andalucia, Sevilla, Consejería de Economía y Hacienda.

- (1995), Encuesta Industrial 1992. Resultados de Andalucia, Sevilla, Ed. Instituto de Estadística de Andalucía.

Martin Rodriguez, M. (Dir.) (1993), Estructura económica de Andalucía, Madrid, Ed. Espasa Calpe.

Román, C. (1987), Sobre el desarrollo económico de Andalucía, Málaga, Ed. Arguval. Indicadores Económicos de Andalucia, varios números. 